\title{
LA INVENCIÓN LINGÜÍSTICA EN LA LITERATURA Y EL CINE EN LOS SIGLOS XX Y XXI
}

\author{
Leticia Gándara Fernández \\ Universidad de Extremadura \\ leticiagf@unex.es
}

\begin{abstract}
Resumen: Este trabajo presenta un estudio descriptivo de los proyectos lingüísticos artificiales construidos para la literatura y el cine en los siglos Xx y Xxi. Por un lado, se establece una aproximación a las lenguas inventadas por J. R. R. Tolkien con el fin de advertir algunos de los principios de su invención lingüística. Por otro, se traza un recorrido por algunos de los proyectos de lenguas artísticas pensados para el ámbito cinematográfico; entre ellos, se analizan el klingon, el na'vi, el dothraki, el valyrio, el pársel y el minionés. Finalmente, este estudio permite observar qué métodos o criterios han utilizado estos inventores de lenguas para elaborar sus esquemas lingüísticos y cómo estos se encuentran irremediablemente influenciados por la cultura a la que se asocian.
\end{abstract}

Palabras clave: lenguas artificiales, lenguas artísticas, J. R. R. Tolkien, literatura, cine

\begin{abstract}
This paper presents a descriptive study of artificial linguistic projects constructed for literature and cinema in the $20^{\text {th }}$ and $21^{\text {st }}$ centuries. On the one hand, it is established an approach to the invented languages of J. R. R. Tolkien in order to observe some of the principles of his linguistic invention. On the other hand, it is traced a journey into some of the projects of artistic languages thought for the cinematographic field; among them, the Klingon, the $N a^{\prime} v i$, the Dothraki, the Valyrio, the Parsel and the Minion are analyzed. Finally, this study allows us to observe what methods or criteria these language inventors have used to elaborate their linguistic schemes and how these are irremediably influenced by the culture to which they are associated.
\end{abstract}

Keywords: artificial languages, artistic languages, J. R. R. Tolkien, literature, cinema

\section{INTRODUCCIÓN}

El interés por la invención de lenguas artificiales surgió en Inglaterra en pleno siglo XVII y estuvo estrechamente relacionado con la búsqueda de una lengua perfecta. El movimiento de creación de sistemas lingüísticos artificiales ha recorrido 
desde entonces todas las etapas de la historia, acompañado inevitablemente de una evolución interna en sus motivaciones y en sus resultados, consecuencia del marco socio-cultural de cada periodo histórico (Martínez, 2016: 78). Los propósitos que guiaron la construcción de los primeros esquemas lingüísticos poco tienen que ver con la finalidad por la que actualmente se siguen diseñando lenguas ${ }^{1}$. En un ejercicio de extrema simplificación, Galán (2018a: 75) establece una clasificación de estos objetivos en tres, que tomamos como modelo para detallar los tipos de lenguas artificiales y las motivaciones que determinaron su creación, así como algunos ejemplos de estos diseños:

A) Motivos científicos o filosóficos, como el «deseo de garantizar la transparencia, racionalidad y univocidad de la comunicación científica mediante nuevos sistemas con proyección universal» (Galán, 2018a: 75). A estos pertenecen las lenguas denominadas filosóficas o a priori (denominación propuesta por Couturat y Leau, 1903) que triunfaron en el siglo XVII, aunque con ejemplos también presentes en los siglos XIX y XX (cfr. Galán, 2012: 421-425). Para su elaboración, los autores no basan su estructura en las lenguas naturales, por considerarlas «instrumentos de comunicación caprichosos, redundantes, ilógicos, irregulares, plagados de ambigüedades, cambiantes e inestables» (Calero, 2010: 18). Autores representativos fueron Pedro Bermudo (1653), Athanasius Kircher (1660), George Dalgarno (1661) y John Wilkins (1668).

B) Motivos pragmáticos, laicos (e incluso filantrópicos, como en el caso del esperanto) que buscan eliminar las fronteras en un espacio internacional (cultura occidental) para favorecer los intercambios comerciales e ideológicos (Galán, 2018a: 75). Estos diseños, denominados a posteriori por Couturat y Leau (1903), toman como base las lenguas naturales, vivas o muertas, e intentan subsanar sus defectos y ambigüedades, con el fin de garantizar un fácil y rápido aprendizaje. El ejemplo más destacado es, sin duda, el esperanto, desarrollado por el oftalmólogo Ludwik Lejzer Zamenhof en 1887 con el fin de asegurar la paz y la unidad entre los pueblos.

c) Motivos artísticos, utópicos, o puramente ficcionales que, partiendo de la idea de que «la perfección de una lengua reside en su capacidad para reflejar especularmente la realidad, pretenden sistemas regulares que sustenten sociedades perfectas y ecuánimes (utopías) en las que las lenguas sean un potente

${ }^{1}$ Para una aproximación a la historia de las lenguas artificiales, véase Couturat y Leau (1903), Eco (1994) y Calero (1999). 
motor del cambio social» (Galán, 2018a: 76). Ejemplos destacados de estas lenguas en la ficción se incluyen en relatos utópicos, tales como Viaje a la Luna (1657) de Cyrano de Bergerac o Los viajes de Gulliver (1726) de Jonathan Swift, o distópicos como Un mundo feliz (1932) de Aldous Huxley, 1984 de George Orwell (1949) o La naranja mecánica (1962) de Anthony Burguess.

En el último grupo de esta clasificación se encuentran también las numerosas propuestas surgidas en los últimos años en el ámbito de la ficción literaria y, sobre todo, cinematográfica; entre otros logros, estos proyectos han conseguido difundir extraordinariamente el fenómeno de la invención de lenguas entre el gran público (Martínez, 2016: 78) y han generado verdaderas comunidades de hablantes en la red (por ejemplo, el grupo Dothraki en Facebook tiene más de 10.000 seguidores y el klingon cuenta con su propio sistema de certificación de nivel en el Instituto de Lengua Klingon).

Estos diseños se denominan «lenguas artísticas», «lenguas ficcionales», «lenguas ficticias», «ideolenguas», «lenguas construidas»o «conlang $\gg^{2}$. Con respecto a su método de construcción, la mayor parte de los inventores de este tipo de lenguas se apunta al método naturalista. Según Jaén (2017: 10), este consiste «en inspirarse en las lenguas naturales e imitar sus complejidades, ambigüedades e irregularidades», algo que no comparten ni con las lenguas a priori ni con las a posteriori, ya que estas persiguen el propósito contrario.

En el marco de las lenguas artificiales, las lenguas artísticas constituyen una categoría aparte. David Crystal (1997: 29) establece que estos sistemas se dividen en: international languages, programming languages, artificial intelligence (AI) y languages and simplified languages. Conforme a esta clasificación, las autoras Lawrie Barnes y Chantelle van Heerden (2006) proponen un quinto grupo para las lenguas ficcionales atendiendo a su función pluridimensional. Destacan que su papel principal dentro de la literatura es el de crear un mundo ficcional. Por un lado, constituyen importantes herramientas con las que generar otros mundos, posibles e imaginarios, de los que forman parte integral; y, por otro, funcionan dentro de dichos universos, conformando contextos sociolingüísticos, en los que constituyen identidades indi-

\footnotetext{
${ }^{2}$ La palabra conlang es la suma de la primera sílaba de los términos ingleses constructed y language. Aparece por primera vez el 29 de julio de 1991 para denominar la lista de correo electrónico en la que se debatían cuestiones relacionadas con la creación de lenguas. Alrededor de este neologismo se crearon otros términos, tales como artlang, loglang, engelang, auxlang jokelang, etc. Para traducir el término conlang al español, Alex Condori propuso en el año 2000 la expresión ideolengua, también como título de una lista de distribución. Sin embargo, aunque actualmente se aceptan las dos acepciones, conlang continúa siendo la opción más utilizada, también en la lengua española. De hecho, esta ha sido aceptada por el Oxford English Dictionary y el Cambridge Dictionary.
} 
viduales y grupales (Barnes y Heerden, 2006: 102). Así mismo, partiendo de la importancia de estas lenguas como conformadoras de mundos en las obras de ficción, Moreno Paz señala que estos representan unidades léxicas que aluden a elementos ficticios en estos universos imaginarios (2016: 817).

J. R. R. Tolkien, por su parte, no considera que el estudio de estas lenguas deba restringirse exclusivamente a su función en los argumentos de ficción, sino que apunta la necesidad de examinar detenidamente la evolución real de estos sistemas. De esta forma, en una de sus cartas de 1967 menciona que las lenguas inventadas tienen dos historias que contar y que, por tanto, ambas deberían tenerse en consideración. La primera de ellas, la «historia externa», se basa en el proceso por el que pasa el autor en la construcción de su sistema lingüístico; es decir, alude, por ejemplo, a la elección de las secuencias sonoras que después se utilizan para forjar los nombres. En cambio, la segunda, la «historia interna», consiste en las propias vivencias imaginarias de sus hablantes, en sus costumbres y culturas, y en cómo las lenguas se desarrollan en dicho contexto de ficción (Tolkien, 1993: 579). Con todo, conocer la «historia externa» de las lenguas — en la realidad - puede ayudar a entender la «historia interna» - en la ficción- de estos diseños lingüísticos.

Ahora bien, para componer la historia interna de estas lenguas es necesario disponer de un profundo conocimiento de lingüística, lo que sobradamente demuestran los creadores de lenguas artísticas. Con respecto a la historia externa, los autores deben poseer una gran imaginación que les permita construir mundos en los que recrear culturas insólitas, que resulten cuanto menos curiosas para sus lectores. No obstante, aunque algunos novelistas de literatura fantástica y de ciencia ficción idean universos paralelos e imaginarios, con lenguas diferentes a las existentes en el mundo real, estos no siempre desarrollan su gramática y su léxico. En muchos casos, tan solo aparecen palabras aisladas o expresiones que se repiten con frecuencia o que tienen cierta relevancia en el argumento. Por ejemplo, en la novela de ciencia ficción Consecuencias naturales (1994) de Elia Barceló se simula cómo una especie de alienígenas habla una lengua artificial, de la que apenas se incluyen vocablos, que consigue establecer un distanciamiento lingüístico entre estos seres y los humanos (Gándara, 2016).

En el ámbito cinematográfico, los encargados de diseñar estos sistemas son lingüistas contratados por las productoras para conseguir determinados efectos en sus filmes. En este caso, las lenguas alcanzan un mayor grado de complejidad y desarrollo debido a que estas se popularizan entre los seguidores de series y películas. Si bien es cierto que el éxito de estas se debe en buena parte a su aparición de en la gran pantalla, pues la recreación de las lenguas inventadas por Tolkien en la adaptación cinematográfica de El señor de los anillos (Jackson, 2001-2003) inauguró una nueva 
era en la historia de las lenguas artificiales diseñadas para el cine. Así, en este trabajo, en primer lugar, realizamos un repaso por algunos de los principios de la actividad lingüística de Tolkien en la creación de lenguas; segundo, destacamos cómo estas traspasan los límites de la ficción literaria y se asientan en los del imaginario cinematográfico. Finalmente, proponemos un recorrido por algunos de los proyectos de lenguas artificiales más importantes construidos para el cine.

\section{LA INVENCIÓN LINGÜÍSTICA EN LA LITERATURA FANTÁSTICA Y DE CIENCIA FICCIÓN}

Las lenguas construidas con propósitos artísticos suelen incluirse en obras pertenecientes a la literatura fantástica o de fantasía y a la ciencia ficción. El primer autor que creó una lengua «completa» para un contexto de ficción literaria fue J. R. R. Tolkien (1892-1973). El filólogo y profesor de la Universidad de Oxford diseñó más de catorce lenguas para sus novelas con el fin de crear un universo fantástico poblado por hombres, orcos, enanos, hobbits, elfos y otras criaturas. Dichas lenguas son el germen del mundo inventado y paralelo que aparece en El hobbit (1937), El señor de los anillos (1954-5) y El Silmarillion (1977). La invención lingüística se postula, por tanto, como el punto de partida, por lo que no resulta extraño que Tolkien señale: «para mí, viene primero el nombre, y luego le sigue la historia» (Tolkien, 1993: 333). El ciclo mitológico que aparece en El señor de los anillos no es más que el deseo de su autor de dar a sus lenguas un «hogar» y criaturas que las hablasen. Afirma, pues, que « las "historias" se crearon más bien para procurar un mundo para las lenguas que a la inversa » (Tolkien, 1993: 333); por lo que no resulta extraño que, además de incluir en el relato abundante material lingüístico, Tolkien confiese:

Habría preferido escribir en «élfico». Pero, por supuesto, una historia como El Señor de los Anillos ha sido preparada para la imprenta y he dejado en ella tanto «lenguaje» como pensé que los lectores podrían digerir. (Compruebo ahora que a muchos les hubiera gustado más). Pero hay abundante material lingüístico (además de nombres y palabras élficas) incluido en el libro o mitológicamente expresado (Tolkien, 1993: 333).

Las historias que Tolkien recrea en la Tierra Media tienen su origen en su pasión por el lenguaje. Como filólogo, mostró siempre una gran admiración por el estudio de las lenguas. Ya en su niñez, su madre le inició al estudio del latín, 
el francés y el alemán. En el colegio, fue poco a poco aprendiendo otras lenguas como el griego, el inglés medio y antiguo, el nórdico antiguo, el gótico y el galés medieval. Aparte de estas, también tenía conocimientos de ruso, sueco, danés, noruego y holandés. Aunque, como el propio autor refiere en sus cartas, los idiomas por los que él sentía una mayor devoción eran el finés y el galés (Tolkien, 1993). Ambos fueron - inevitablemente - su fuente de inspiración para la invención de las lenguas élficas ${ }^{3}$.

Aunque Tolkien dedicó buena parte de su vida a la construcción de lenguas artificiales, este mantuvo su afición por la invención lingüística casi como un secreto, que tan solo descubrió en una conferencia en $1931^{4}$. Dicho relato se conservó en un manuscrito titulado "Un vicio secreto", publicado póstumamente como parte de la obra Los monstruos y los criticos y otros ensayos (1983). Christopher Tolkien, hijo del autor, considera este ensayo una pieza única «en el sentido de que tan solo en esta ocasión singular -según parece- apareció en público y por derecho propio el "mundo inventado" ante el "mundo académico"» (Tolkien 1998: 7).

Tolkien ideó estos modelos lingüísticos con el fin de satisfacer sus propios gustos personales, especialmente en materia lingüística. De este modo, afirma «estas "cosas" fueron construidas expresamente para ser únicamente personales, y para proporcionar una satisfacción privada, no a modo de experimento científico, ni mucho menos aún con la esperanza de tener una audiencia $\gg$ (Tolkien, 1998: 254). La suya fue, principalmente, una motivación lingüística, como bien afirma en el prólogo a la segunda edición de El señor de los anillos: «primarily linguistic in inspiration and was begun to provide the necessary background of 'history' for Elvish tongues» (Tolkien, 1965: 7).

En consecuencia, el filólogo elaboró un grupo de lenguas autoconsistentes y técnicamente convincentes, documentando incluso su origen, evolución y desa-

\footnotetext{
${ }^{3}$ Algunos autores señalaban que su gusto por las lenguas nórdicas, a las que Tolkien prefería denominar germánicas, provenía exclusivamente de su madre, quien sabía alemán y le dio las primeras lecciones en esa lengua. Además, él mismo menciona en una carta que a su madre «también le interesaba la etimología y despertó mi propio interés por ella; y también por los alfabetos y las escrituras» (Tolkien, 1993: 571).

${ }^{4}$ El título Un vicio secreto fue aprobado por Christopher Tolkien. El título originario del manuscrito es Un pasatiempo hogareño (con una nota posterior: «En otras palabras: idiomas hechos en casa o inventados»). Sin embargo, en una carta de 1967, Tolkien hace referencia a su ensayo con este título: «El pasatiempo de inventar idiomas es muy común entre los niños (una vez escribí un estudio sobre eso, titulado Un vicio secreto)» (Tolkien, 1993: 569). De Un vicio secreto existe un único manuscrito, sin fecha ni indicación alguna de la ocasión para la que fue preparada; pero resulta evidente que el auditorio era una sociedad filológica, y el congreso de esperanto en Oxford al que se hace referencia al principio del ensayo como ocurrido «hace más o menos un año» tuvo lugar en julio de 1930. Así que podemos fijar la fecha en 1931 (Tolkien, 1998: 10).
} 
rrollo en un mundo ficticio en el que se adaptaban perfectamente, con palabras, expresiones y breves fragmentos. Para que estas lenguas sobreviviesen, su creador se encargó de darles una audiencia. El profesor estaba completamente convencido de que sus lenguas necesitaban personas o, más bien, criaturas que las hablasen. Solo de esta forma, sus lenguas podrían desarrollarse en un contexto de ficción de idéntica forma a como lo hacen las lenguas naturales en el mundo real. Con este fin, esquematiza detalladamente las relaciones históricas y los cambios que experimentan sus lenguas con el paso del tiempo (Hostetter, 2007: 334). Un hecho que no solo le permite caracterizarlas individualmente, sino también trazar diferenciaciones entre ellas.

Este último aspecto se considera uno de los rasgos fundamentales de la producción lingüística de Tolkien, hasta el punto de que algunos autores lo han calificado como el núcleo de su invención (Smith, 2014: 204). Y es que esta distinción permite, por ejemplo, contrastar la belleza lingüística de las lenguas élficas, pensadas para trasmitir armonía y agrado, con la lengua negra, prototipo de la fealdad lingüística, por ser intrínsecamente desagradable (Shippeys, 2000: xiv). Estos contrastes evidencian, además, la estrecha relación existente entre lengua y cultura en su obra, pues mientras los elfos cuidan de su lengua como si de una obra de arte se tratara, los orcos descuidan su lenguaje, deformando las palabras y expresiones propias del oestron o lengua común 5 .

Las lenguas élficas, concretamente el quenya y el sindarin, son las más elaboradas dentro del legendarium de Tolkien. La primera se caracteriza por ser una lengua antigua, una especie de «latín élfico», utilizada por los elfos en ceremonias y en asuntos de canto y de ciencia. En cambio, el sindarin o élfico gris es la lengua viva que emplean estos sujetos para comunicarse. A ella pertenecen la mayor parte de los nombres de lugares y seres que encontramos en El señor de los anillos, tales como Baranduin, Elbereth 'hacedora de las estrellas', Gondolin 'la Roca Escondida', etc.

Aunque el quenya y el sindarin constituyen diseños lingüísticos diferentes, ambos derivan de una lengua común; de hecho, Tolkien declara haber introducido deliberadamente una serie de cambios para «darle un carácter lingüístico muy semejante (aunque no idéntico) al británico-galés» (Tolkien, 1993: 274). Según el autor, dichas modificaciones obedecen a dos razones: «porque encuentro ese carácter muy atractivo a algunos temples lingüísticos, y porque parece adecuarse al tipo de leyendas o historias más bien "célticas" que cuentan sus hablantes» (Tolkien,

${ }^{5}$ Un ejemplo lo encontramos en el Apéndice F: «En su jerga, tark [tarco], "hombre de Gondor, era una forma corrompida de tarkil, palabra quenya utilizada en oestron para designar a quien tuviera ascendencia Númenóreana» (Tolkien, 1987: 130). 
1993: 274). De tal forma, el sindarin presenta una gran influencia del galés mientras que en quenya aparecen rasgos propios del finés.

La influencia del finés en el quenya se observa principalmente en la estructura de los nombres; estos últimos caracterizados por la ausencia de género, sus cuatro números y su carácter declinativo. Al igual que el latín y el finés, el quenya presenta casos gramaticales, que a su vez reflejan rasgos propios de esta lengua nórdica. Entre otros aspectos, en quenya el caso locativo presenta un valor idéntico al del finés, pues en ambas lenguas se utiliza como equivalente del verbo «tener»; el ablativo significa 'de parte de quién' en ambos sistemas; y el alativo puede emplearse como complemento circunstancial de finalidad y como complemento indirecto en la oración (cfr. González, 2002). El influjo del finés se observa también en los sufijos posesivos, agregados al nombre después de la inflexión preposicional (atari-nya 'mi padre') y en el uso de preposiciones y posposiciones, como en pella 'más allá de'.

Por otro lado, el sindarin, lengua paratáctica con escasas inflexiones, toma del galés el fenómeno de la lenición o mutación suave, ya esta se produce en casos semejantes a los de la lengua celta, concretamente en posición intervocálica (Hostetter, 2007: 6). Uno de los ejemplos más destacados se encuentra en el fragmento más extenso de sindarin conservado, conocido como «La Carta del Rey», en la que Aragorn informa a Sam sobre sus planes de visitar la Comarca. En su carta, Aragorn escribe: Aragorn Arathornion Edhelham anglenneath a IVaranduiniant erin dolothen Ethuil ( «Aragorn, hijo de Arathorn, Piedra de Elfo, se aproximará al puente del Baranduin el octavo día de la Primavera»). La lenición es provocada por $i$ (artículo) en la palabra Varanduinaiant donde $v$ se suaviza en $b$ dando lugar al término Baranduiniant, cuya traducción es 'sobre el río Baranduin (Brandivino)' (Salo, 2004: 225-6).

La producción artística de Tolkien, además de ser objeto de estudio en diferentes disciplinas, se ha convertido también en una inagotable fuente de inspiración para los inventores de lenguas de décadas posteriores, especialmente para aquellos lingüistas que diseñan lenguas para el ámbito cinematográfico. El estudio de estos proyectos, que han tenido una gran repercusión en la actualidad, nos ocupa en el siguiente apartado.

\section{LA INVENCIÓN LINGÜÍSTICA EN EL CINE EN LOS SIGLOS XX Y XXI}

La aparición de las creaciones lingüísticas de Tolkien en la adaptación cinematográfica de El señor de los anillos supone un hito importante dentro del movimiento de creación de lenguas artificiales. Desde entonces, construir una lengua se ha convertido 
en casi una obligación para todos aquellos que deseen recrear universos imaginarios en sus filmes cinematográficos o series televisivas ${ }^{6}$. Generalmente, estas lenguas son tan insólitas y sorprendentes como las asombrosas y extrañas culturas a las que complementan. Este hecho permite, según Lozano (citado en Robles, 2016), «potenciar la identificación del espectador con lo atractivo y lo exótico de razas, culturas y civilizaciones que parecen tan reales como la suya propia ». Por tanto, además de aportar una mayor dosis de verosimilitud a esos mundos paralelos, consiguen despertar la curiosidad de su público. En líneas generales, y salvo excepciones, los creadores de lenguas de las últimas décadas persiguen que sus modelos sean diferentes de los paradigmas lingüísticos europeos; para ello suelen acudir a lenguas minoritarias o de origen incierto, como el vasco, el tagalo o el azteca (Galán, 2009: 127). Por ejemplo, el pársel, inventado por Nolan para Harry Potter (1997-2007), presenta la ergatividad del euskera y el orden de palabras V-S-O del tagalo (Gándara, 2018a: 167).

Como afirma Lozano (citado en Robles, 2016), los conlangs no son solo instrumentos con los que expandir la experiencia ficcional, sino también contenidos que nos permiten abrir la puerta a numerosas posibilidades de participación con la audiencia. Pues la aparición de lenguas artificiales en el cine origina nuevos retos tanto para actores como para espectadores. Mientras que los primeros afrontan la ardua tarea de aprender y memorizar lenguas radicalmente distintas a las suyas; los segundos se enfrentan a la lectura de subtítulos. Este último aspecto es, según Peterson (2015a: 34), un auténtico milagro, pues implica que millones de personas se muestren partidarias de la lectura de subtítulos, algo a lo que el espectador no siempre está dispuesto.

A mediados del siglo xx, cuando se inicia este movimiento, las películas y series empleaban simplemente un vocabulario diseñado ad hoc para los hablantes de

${ }^{6}$ También encontramos lenguas artificiales en los videojuegos y en la música. Notables contribuciones artísticas son, por ejemplo, los diseños de M.A.R. Barker para los juegos de rol Empire of the Petal Throne (1974-5). En los videojuegos, destaca el simlish o lengua de los sims (1996); en este caso, la intención de su autor fue ahorrar en gastos de traducción y evitar que las frases que articulan los sims en el juego fuesen demasiado repetitivas. Otro ejemplo aparece en The Longest Journey (1999), basado en una aventura gráfica en la que el participante debe averiguar el significado de los diferentes enigmas que se plantean para lograr avanzar en el juego. Dichos enigmas aparecen en una lengua artificial denominada alltongue. Como ambientación, también se utilizan otros códigos lingüísticos en Ico (2001) y Shadow of the Colossus (2018). Por otro lado, en la música, citamos el caso del kobaïano (1969). Se trata de una lengua creada por el batería y compositor francés Christian Vander para su banda de rock Magma. El kobaïano es la lengua de Kobaïa, un planeta ficticio inventado por Vander para una "ópera espacial” cantada en esta lengua construida. Otra creación lingüística en este medio es el loxian (2005), lengua artificial creada por la escritora y letrista Roma Ryan para el álbum Amarantine (2005) de Enya. El loxian aparece en tres canciones de este álbum: "Less than a Pearl", "The River Sings" y "Water Shows the Hidden Heart". 
lugares ficticios (primeros episodios de Danger Man 1960-68) o para las lenguas que los cineastas no querían reproducir fielmente (Thoroughly Modern Millie 1967). En este momento, el crear una lengua completa para una nación ficticia era algo impensable. De hecho, la primera vez que aparece un diseño inventado en una serie de televisión fue en Land of the Lost (1974); en esta, se incluía una lengua llamada paku o pakuni para una raza entre humano y primate denominados con este mismo nombre. Este hecho cobra cierta importancia por dos motivos. En primer lugar, porque se trata de una lengua inventada por una mujer, Victoria Fromkin (1923-2000), lingüista y profesora en la Universidad de California en los Ángeles. Algo que supone un hito en el movimiento de lenguas artificiales ya que las mujeres representan paradójicamente una clara minoría en este ámbito, pese a ser pioneras en el mismo. No olvidemos que la primera lengua artificial documentada, la Lingua Ignota, fue construida por la abadesa Hildegard von Bingen en el siglo XII (cfr. Galán, 2018b). En segundo lugar, porque Fromkin fue la primera lingüista contratada para crear una lengua para un contexto de ficción. Se inicia así una era en la que cada vez es más frecuente que las productoras contraten a profesionales del lenguaje para la invención de lenguas para el cine.

De entre las muchas lenguas construidas para este ámbito, el klingon es uno de los proyectos lingüísticos que más éxito ha tenido. El nombre de la lengua se corresponde con el de una raza de humanoides del universo de Star Trek, los klingons. Esta se menciona por primera vez el 23 de marzo de 1967 en «Errand of Mercy», un episodio de esta serie televisiva. La segunda alusión al klingon se registra en un episodio posterior de este mismo año, «The Trouble with Tribbes», cuando uno de los personajes alude a que parte de los habitantes de su galaxia están aprendiendo a hablar klingonese. No será hasta el estreno de la primera película, Star Trek: The Motion Picture (1979) cuando aparezcan algunos diálogos en la lengua de los klingons. El encargado de esbozar estas primeras palabras fue James Doohan, el actor que protagonizaba al personaje Montgomery Scott en la serie. El klingon reaparecerá en la tercera película, Star Trek III: The Search for Spock (1984); aunque para esta producción ya se decidió que los klingons deberían tener su propia lengua. Esta fue construida por el lingüista Marc Okrand (1948). En un principio, no se pensó en crear una lengua completa sino solo aquellos términos necesarios para que los personajes pudieran establecer diálogos en klingon. Más adelante, dada la aceptación que tuvo entre sus seguidores, se consideró conveniente dotar a este sistema de una gramática y un vocabulario propios, que se publicaron posteriormente en diferentes manuales y diccionarios como The Klingon Dictionary (1992). Su propósito no era otro que el de idear un sistema lingüístico cuyo sonido fuese diferente a todas las lenguas naturales. Con el fin de dotar a esta lengua de un aspecto alienígena, el klingon presenta 
el orden de palabras O-V-S (Objeto-Verbo-Sujeto) y una gran variedad de sonidos graves y guturales, similares a gruñidos. Además, se trata de una lengua aglutinante, que utiliza afijos para variar el significado de los términos y cuyo léxico se encuentra estrechamente relacionado con el mundo de la guerra y los insultos ${ }^{7}$. Algunos de estos son: P'atagh! 'pedazo de mierda'; lo'Be Vos, 'cobarde'; Hab SoSLI'Quch!, 'tu madre tiene la frente lisa'; o QI'yaH!, intraducible por ser el peor insulto en klingon.

Si el fuerte carácter de los klingons exigía una lengua que denotara dureza, el apacible talante de los na'vis requería un sistema con un sonido agradable. Este proyecto, que el lingüista Paul R. Frommer comenzó a elaborar en 2005, está pensado para los asombrosos seres humanoides de piel azul que habitan en la luna de Pandora en la película Avatar (dirigida por Cameron en 2009). La finalidad de esta lengua no es otra que transmitir armonía y belleza al ser escuchada, algo que no resulta extraño si tenemos en cuenta que los návis aparecen caracterizados positivamente. Dado que se trata de una raza alienígena, dicho sistema presenta también una gran complejidad, pues se pretendía que fuese diferente al resto de lenguas naturales. Algunos de sus rasgos son: un sistema de siete vocales $(a, \ddot{a}, e, i, i, o, u)$; la presencia de consonantes eyectivas $(/ \mathrm{p} \rrbracket, \mathrm{t} \rrbracket, \mathrm{k} \bigotimes /)^{8}$; la aparición de complejos grupos consonánticos, como en fngap, cuyo significado es 'metal'; y la posibilidad de que $l l \mathrm{y} r r$ funcionen como núcleos silábicos, como en plltxe 'hablar'. A diferencia de la mayor parte de las lenguas naturales, distingue cuatro géneros gramaticales (masculino, femenino, común y neutro) y su sistema de casos engloba el ergativo, característico del euskera? ${ }^{9}$.

En la misma línea que las anteriores se encuentran las creaciones lingüísticas elaboradas para la serie televisiva Juego de tronos (2011), basada en las novelas de fantasía épica Canción de bielo y fuego (1996) de George R. R. Martin (1948). Este autor no fue el encargado de elaborar las lenguas que hablan los habitantes de los Siete Reinos de Essos y Poniente, sino que se limitó a incluir una serie de palabras, principalmente nombres propios, que serán de gran utilidad para el inventor de los idiomas (cfr. Gándara, 2018a: 201). Este hecho explica que no encontremos diálogos completos en este tipo de lenguas en las novelas, pero sí puedan escucharse en su adaptación televisiva. El encargado de diseñar estos códigos fue el lingüista estadounidense David J. Peterson (1981).

El dothraki es la lengua propia de los dothraki, un pueblo de nómadas guerreros

\footnotetext{
${ }^{7}$ Galán destaca que el klingon es una «mezcla de yiddish (ingrediente que hace referencia a la antigüedad adámica) y de japonés» (2007: 66).

${ }^{8}$ Aquellas en las que "el aire queda comprimido entre la glotis cerrada y el lugar de articulación en el tracto bucal y sale al exterior por un movimiento ascendente de la laringe" (Llisterri, 2018).

${ }^{9}$ Los principales rasgos gramaticales y léxicos del Na'vi se encuentran en el siguiente enlace: https://learnnavi.org/.
} 
que habitan en las llanuras del Mar Dothraki, en el continente de Essos. El nombre de la lengua coincide, por tanto, con el del pueblo dothraki, cuyo significado literal es men who ride, the Dothraki people (Littaver, 2016: 23). Para la elaboración de este sistema, Peterson parte de las 56 palabras que ya había incluido Martin en las novelas. Sus esfuerzos se encaminaron a la creación de una lengua que, además de provocar cierta extrañeza al ser escuchada, denotara dureza (Peterson, 2015b: 25) ${ }^{10}$.

El dothraki presenta un total de 27 fonemas consonánticos, entre los que no se incluyen bilabiales oclusivas ni sordas ni sonoras. Su inventario es el siguiente:

\begin{tabular}{|l|l|l|l|l|l|l|l|}
\hline & Labial & Dental & Alveolar & PALATAL & Velar & Uvular & Glotal \\
\hline Oclusiva & & $\mathrm{t}, \mathrm{d}$ & & & $\mathrm{k}, \mathrm{g}$ & $\mathrm{q}$ & \\
\hline Africada & & & & $\mathrm{d} 3,\left(\mathrm{t} \int\right)$ & & & \\
\hline Fricativa & $\mathrm{f}, \mathrm{v}$ & $\mathrm{\theta}$ & $\mathrm{s}, \mathrm{z}$ & $\int, 3$ & $\mathrm{x}$ & & $\mathrm{h}$ \\
\hline Nasal & $\mathrm{m}$ & $\mathrm{n}$ & $\mathrm{n}$ & $\mathrm{n}$ & $\mathrm{\eta}$ & $\mathrm{N}$ & \\
\hline Aproximante & & & & $\mathrm{j}$ & $\mathrm{w}$ & & \\
\hline Lateral & & $\mathrm{l}$ & & & & & \\
\hline Vibrante & & & $\mathrm{f}, \mathrm{r}$ & & & & \\
\hline
\end{tabular}

Tabla 1. Inventario fonológico (consonantes) del dothraki (Peterson, 2015b: 92)

\begin{tabular}{|l|l|l|}
\hline & \multicolumn{1}{|c|}{ ANTERIOR } & \multicolumn{1}{c|}{ POSTERIOR } \\
\hline Cerrada & $\mathrm{i}$ & \\
\hline Intermedia & $\mathrm{e}$ & $\mathrm{o}$ \\
\hline Abierta & & $\mathrm{a}$ \\
\hline
\end{tabular}

Tabla 2. Inventario fonológico (vocales) del dothraki (Peterson, 2015b: 92)

Como puede apreciarse en las tablas anteriores, solo aparecen cuatro fonemas vocálicos: /a/, /e/, /i/ y /o/. El único elemento vocálico que no se utiliza es /u/; tan solo se registra en el grupo qu-por iniciativa de Martin, como en 2 uaro y Jhiqui (nombres propios). Del mismo modo, destacan una serie de rasgos que conforman la esencia de este diseño: la fricativa velar sorda $[\mathrm{x}]$ (en khal 'jefe' o arakh 'espada curvada', símbolo dothraki), la vibrante múltiple [r] (en khalasar 'horda dothraki' o lajasar 'ejército'), la oclusiva uvular sorda [q] (en qoy 'sangre' o qora 'brazo'), la fricativa glotal [h] (que con frecuencia aparece como [h]) (en hrazef'caballo' o mahrazh 'hombre') y las consonantes dobles y geminadas (en iffi 'victoria' o allayafat 'complacer'). El autor de la lengua se asegura de que estos sonidos aparezcan asiduamente en

${ }^{10}$ En 2014 se publicó el curso Living Language Dothraki, creado por David J. Peterson, en el que se incluye información referente a la gramática y el léxico de esta lengua. 
su lengua mediante el uso de una serie de estrategias, entre las que destaca la adición de afijos. Por ejemplo, Peterson convierte la fricativa velar sorda $[\mathrm{x}]$, representada como $k h$, en el sufijo derivativo -i (kh), que da lugar a términos como kachrakh 'oler', nesikh 'conocimiento', sewafikh 'vino', etc. (Gándara, 2018b: 320).

Para Juego de tronos, Peterson también elaboró el alto valyrio, lengua de la Antigua Ciudad de Valyria, que contribuye a la representación de un imperio derruido, ya que se asocia a la última superviviente de una casa nobiliaria que fue completamente devastada, Daenerys Targaryen. En las novelas, se introducen algunas palabras y expresiones, tales como valar morghulis 'todos los hombres deben morir', valar dohaeris 'todos los hombres deben servir' o dracarys 'fuego de dragón'. De nuevo, Peterson se basó en estos términos para establecer los principales rasgos gramaticales y léxicos de su conlang. Este se caracteriza por presentar una amplia variedad de nasales y de alófonos para distintos fonemas con el fin de mostrar un estado de evolución en la lengua. Su sistema vocálico distingue entre cortas y largas; estas últimas marcadas con un macrón: ā [a : ], a [a]; e [e : ], e [e]; ō [o : ], o [o]; ù [u : ], u [u]. El sistema de número se amplía a singular, plural, paucal (para indicar 'varios', 'unos cuantos'; aparece en hopi, ruso y árabe) y colectivo (designa un grupo como una unidad; característico del griego arcaico y el japonés). Como ejemplo, citamos: vala 'hombre'; vali 'hombres'; valum 'algunos hombres'; y valar 'todos los hombres'. Nombres y adjetivos se dividen en tres clases, que presentan diferentes terminaciones en función de los casos gramaticales, y en cuatro géneros basados en los elementos de la naturaleza: solar, lunar, terrestre y acuático (cfr. Gándara, 2018a).

Igualmente, hay también ciertos diseños lingüísticos que no adquieren el estatus de lengua. Algunos autores coinciden en denominar relex a estos sistemas, puesto que, aunque no se consideran «lenguas», se crean mediante el uso de reglas lingüísticas reales (Olivera, 2015: 58) ${ }^{11}$. En este cajón de sastre se incluyen construcciones lingüísticas como el pársel o lengua de serpientes de la saga Harry Potter y la lengua de los minions, entre otras. El primero no fue desarrollado por J. K. Rowling, autora de la saga Harry Potter (1997-2007), para su inclusión en esta obra literaria; en los libros, los encuentros entre Harry y las serpientes se describen siempre en lengua inglesa. No hay, por tanto, ninguna muestra de pársel en las novelas, a diferencia de lo que sucede en las adaptaciones cinematográficas, en las que sí es posible escucharlo. En concreto, aparece en Harry Potter y la cámara secreta (2002), Harry Potter y el cáliz de fuego (2005) y las dos partes de Harry Potter y las reliquias de la muerte (2010, 2011). El encargado de desarrollar los rasgos fonéticos y gramaticales que caracterizan a este

${ }^{11}$ El término relex es un acortamiento de relexificación o proceso de sustitución del vocabulario de una lengua. En el contexto de las lenguas artificiales, este se utiliza en casos en los que se mantiene la gramática de una lengua natural, pero se sustituye su vocabulario por uno de creación propia. 
código de serpientes fue Francis Nolan, profesor en la Universidad de Cambridge. Nolan se enfrentó a un auténtico reto en la construcción de este sistema. El pársel debía: 1) emular los silbidos que emiten las serpientes; 2) ser apropiado y "fácil" de pronunciar para los actores; 3) denotar cierta desconfianza y terror al ser escuchado. Su finalidad era que todo aquel que no dominase la lengua solo fuese capaz de escuchar silbidos. Para conseguir este objetivo, Nolan prestó especial atención a la fonética. De tal forma, encontramos un sistema de tres vocales $(a, e, i)$, con un gran predominio de la vocal abierta $/ a /$. En cuanto a las consonantes, hay una gran presencia de fricativas, entre las que destacan la fricativa dental sorda $/ \theta /$ (representa th en inglés), la fricativa postalveolar sorda $[S]$ (como en caixa en portugués o en shower en inglés) y, especialmente, la fricativa velar sorda $/ \mathrm{s} /$. Esta última puede aparecer incluso duplicada o triplicada. Por otra parte, no hay fonemas bilabiales y sí una gran presencia de consonantes dobles y geminadas, con el fin de alargar lo máximo posible la pronunciación de los sonidos. Se trata de una lengua tipo V-S-O (Verbo-Sujeto-Objeto), orden de palabras propio del árabe, el tágalo y el galés. Finalmente, conviene destacar la importancia del susurro (o pronunciación parentética en voz baja) como elemento fundamental en el diseño de este sistema. Pues, según Nolan, solo si modificamos la voz y pronunciamos con una intensidad mínima, lograremos el efecto deseado en pársel.

Un caso diferente en el marco de las lenguas creadas con propósitos artísticos es el minionés, idioma banana o Minion Gibberish ('discurso incomprensible o sin sentido'), compuesto por Pierre Coffin (1967), uno de los directores de las tres películas de Gru: Mi villano favorito $(2010,2013,2017)$ y el spin-off Los Minions (2015). Los minions son pequeños seres amarillos que han existido desde siempre y que se caracterizan por haber servido a amos de todas partes del mundo ${ }^{12}$. Por ello, tiene sentido que su idioma sea una combinación de palabras internacionales, algo que afecta lingüísticamente a su cultura, pues su capacidad de habla les permite tener un comportamiento similar al de los humanos (Manoharan y Sang Jones, 2015: 2). El hecho de que las palabras recuerden fonéticamente a las lenguas naturales se debe a que se trata de un sistema basado en la deformación de términos del francés, español, inglés, italiano, coreano, japonés y filipino. Por este motivo, no resulta difícil entender algunos de los términos que utilizan en sus diálogos.

\section{CONCLUSIÓN}

Este estudio nos ha permitido observar cómo la invención lingüística ha quedado relegada en la actualidad al terreno de lo estético y al ámbito de los mundos

${ }^{12} \mathrm{El}$ término minion proviene del inglés y significa 'secuaz, subalterno'. De ahí que se emplee para designar a estos personajes ficticios cuya debilidad es servir a los mayores villanos. 
ficticios llevados al cine (Galán, 2018b: 13). Para los constructores de lenguas de las últimas décadas, Tolkien constituye un auténtico referente tanto para el diseño de los rasgos gramaticales y léxicos de sus lenguas como para la construcción de su imaginario lingüístico. De hecho, George R. R. Martin define a Peterson como uno de los herederos de Tolkien:

I really have to give David Peterson 95 percent of the credit on the languages. Tolkien was a world-class linguistic, and even before has writing The Lord of the Rings, he created not one but two elvish languages. He also created the language of the dwarves, Westernesse, and Numenor -he loved creating fantasy languages. His true heir, in that sense, is absolutely David Peterson (Taylor, 2014: 17).

La influencia de Tolkien es evidente, por ejemplo, en el alto valyrio de Peterson, que recuerda sutilmente al quenya. La lengua de Juego de tronos se presenta como una especie de alto élfico, una lengua muerta conservada en manuscritos, que da origen a una extendida familia de lenguas, denominada lenguas valyrias, basada en las lenguas indoeuropeas; esta recuerda al árbol lingüístico que Tolkien desarrolló en sus obras mediante el esquema habitual de representación del parentesco de lenguas del siglo XIX.

En suma, conviene establecer una clara distinción entre las creaciones lingüísticas de Tolkien y el resto de lenguas artísticas posteriores. Mientras las primeras responden a una motivación lingüística, las segundas obedecen a razones artísticas solo en segundo lugar. Por ello, es posible afirmar que estos últimos diseños constituyen soluciones a problemas artísticos, no lingüísticos (Gándara, 2018a: 311). Esto nos permite, además, trazar una diferenciación entre ambos sistemas, no solo por su método de construcción, sino también por su capacidad para generar culturas o adaptarse a ellas.

En la obra literaria de Tolkien se observa una estrecha conexión entre lengua y cultura; ambas diseñadas minuciosamente por parte de su autor. Algo que no ocurre en el resto de lenguas inventadas posteriormente para el ámbito cinematográfico, en el que los autores deben construir sus lenguas para culturas impuestas. Este hecho conlleva que, en algunas ocasiones, presten mayor atención a asociar su invención lingüística a una determinada cultura, más que al propio diseño de la primera.

Las lenguas construidas desempeñan diversas funciones en las culturas en las que se integran. Uno de sus principales cometidos es complementar los universos ficcionales de los que forman parte, en los que deben funcionar de manera idéntica a como lo hace una lengua natural en el mundo real. Dentro de este ámbito ficcio- 
nal, estos sistemas pueden contribuir a la construcción de entidades individuales o grupales. Con respecto al primer caso, entre las lenguas mencionadas en este trabajo, destaca el caso del pársel en Harry Potter. Aunque no se trata de una lengua completa, su inclusión en los filmes refuerza la caracterización del personaje de Lord Voldemort y ayuda a la configuración de un entramado cultural estrechamente vinculado a las serpientes. En cuanto a las identidades grupales, en el caso del klingon y el návi, las lenguas construidas facilitan la conformación de colectivos alienígenas. Mientras que la primera permite distinguir a los klingon como una raza guerrera distinta al resto de seres del universo de Star Trek, la segunda diferencia de los humanos a los enormes seres de piel azul que habitan en la luna de Pandora. En ambos casos, dichas especies alienígenas no solo poseen sus respectivas costumbres y creencias, sino también sus propias lenguas.

Finalmente, en el caso del dothraki, observamos cómo dicha lengua se convierte en un rasgo distintivo de un pueblo salvaje dentro del universo ficticio recreado en Juego de tronos. Su vínculo con el mundo de los equinos, el saqueo y la violencia implica que su lengua albergue un gran número de términos para referirse a los caballos, a las armas y a la guerra. En contraposición, el alto valyrio se asocia a la última superviviente de una casa nobiliaria; por ello, se pensó en un sistema cuyo aspecto evocase una verdadera lengua antigua. Las diferentes variedades dialectales, que conforman la familia de las lenguas valyrias, que muestran las Nueve Ciudades Libres del continente de Essos no solo originan una diversidad lingüística en un contenido de ficción, sino que representan los últimos vestigios de lo que tiempo atrás fue un gran imperio.

En resumen, el hecho de que los autores se muestren más interesados en establecer asociaciones entre la lengua y la cultura impuesta en las obras que en la propia elaboración del sistema lingüístico supone que estos descuiden voluntaria o involuntariamente su proceso de creación. Una imprudencia que a menudo da lugar a ciertas irregularidades y a una falta de sistematicidad evidente en la construcción de los rasgos gramaticales y léxicos de estas lenguas. Esto contrasta con las cuidadas creaciones lingüísticas de Tolkien; pues aunque suponen el origen del movimiento de creación de lenguas artísticas, en absoluto se asemejan a los proyectos de lenguas con fines estéticos de los siglos XX y XXI.

\section{BIBLIOGRAFÍA}

Barnes, Lawrie y Heerden van HeErden (2006): «Virtual languages in science fiction and fantasy literature», Language Matters, 37, 1, pp. 102-117. 
Calero Vaquera, María Luisa (1999): Proyectos de lengua universal. La contribución española, Córdoba, Publicaciones de la Universidad de Córdoba y Obra Social y Cultural Cajasur.

Calero Vaquera, María Luisa (2010): «Las irregularidades lingüísticas desde la perspectiva de los inventores de lenguas universales», en Carsten Sinner y Alfonso Zamorano (eds.), La excepción en la gramática española. Perspectivas de análisis. (Lingüística Iberoamericana 41), Madrid y Frankfurt, Iberoamericana Editorial Vervuert, pp. 15-34.

Couturat, Louis y Léopold Leau (1903): Histoire de la langue universelle, Hildesheim, Georg Olms Verlag.

Crystal, David (1997): A dictionary of linguistics and phonetics, Oxford, Blackwe11 .

Eco, Umberto (1994): La búsqueda de la lengua perfecta, Barcelona, Crítica.

Galán Rodríguez, Carmen (2007): «Imago mundi: las lenguas imaginarias de la ciencia ficción », en Ramón Sarmiento y Fernando Vilches (coords.), Neologismos y sociedad del conocimiento. Funciones de la lengua en la era de la globalización, Madrid, Fundación Telefónica y Editorial Ariel, pp. 59-69.

Galán Rodríguez, Carmen (2009): «La invención de lenguas en la ficción literaria », Investigaciones lingüisticas en el siglo XXI, 3, pp. 103-129.

Galán RodrígueZ, Carmen (2012): «Lenguas universales», en Alfonso Zamorano Aguilar (coord.), Reflexiones lingüisticas en la España del XIX. Marcos, panoramas y nuevas aportaciones, Munich, Lincom Europa, pp. 417-442.

Galán Rodríguez, Carmen (2018a): «Género, sexo y lenguas artificiales», BSEHL, 12, pp. 75-93.

Galán Rodríguez, Carmen (2018b): Glosolalias femeninas e invención de lenguas, Córdoba, UCOPress.

GÁNDARA FERnÁNDEZ, Leticia (2016): «Análisis de los procedimientos lingüísticos en Consecuencias naturales de Elia Barceló», Anuario de Estudios Filológicos, XXXIX, pp. 79-90.

Gándara FERnÁndez, Leticia (2018a): El imaginario lingüístico en la literatura y en el cine. Del élfico al dothraki. Cáceres: Universidad de Extremadura.

Gándara Fernández, Leticia (2018b): «Introducción a la fonética del dothraki, lengua de Juego de tronos», Estudios Humanisticos. Filologia, 40, pp. 309-326, DOI: 10.18002/ehf.

GonzÁlez BAIXAUli, Luis (2002): La lengua de los elfos. Una gramática para el quenya de J. R. R. Tolkien: Tengwesta Kwenyava, Barcelona, Minotauro.

JaÉn MARTín, Miguel (2017): Cómo crear una lengua. Manual para elaborar un idioma propio, Córdoba, Berenice. 
Hostetter, Carl (2007): «Invented languages by Tolkien», en Michael C. D. Drout (ed.),J. R. R. Tolkien Encyclopedia: Scholarship and Critical Assessment, Nueva York, Routledge Taylor and Francis Group, pp. 332-344.

Littaver, Richard (2016): The Dothraki Language Dictionary. En línea: <https:// docs.dothraki.org/Dothraki.pdf> [02/01/2019].

LLISTERRI, Joaquim (2018): La clasificación articulatoria de los sonidos del habla. En línea: <http://liceu.uab.es/ joaquim/phonetics/fon_produccio/clasificacion_articulatoria.html\#Eyectivas $>$ [26/02/2018].

Manoharan, Krisho y Ruth SAng Jones (2015): «Unravelling the Minion Genome», Journal of Interdisciplinary Science Topics, 40, pp. 1-3.

Martínez Gavilán, María Dolores (2016): «La contribución de Caramuel a la creación de lenguas artificiales: características universales, lenguas filosóficas y lenguas secretas», Revista de Investigación Lingüistica, 19, pp. 77-106.

Moreno Paz, María del Carmen (2016): «La traducción de elementos ficticios en The Hobbit (1937) de J. R. R. Tolkien», Revista académica liLETRAd, 2, pp. 213-824.

Okrand, Marc (1992): The Klingon Dictionary. English/Klingon, Klingon/English. The official guide to Klingon words and phrases, Nueva York, Pocket Books.

Olivera, Daniel (2015): «El arte élfico de la lingüística. Tolkien y su relación con el lenguaje y las lenguas artificiales», Estel, 84, pp. 51-66.

Peterson, David J. (2014): Living Language Dothraki, Nueva York, IVY Books.

Peterson, David J. (2015a): «The Languages of Ice and Fire», en Jes Battis y Susan Johnston (eds.), Mastering the Game of Thrones: Essays on George R.R. Martin's A Song of Ice and Fire, Jefferson. McFarland, pp. 15-34.

Peterson, David J. (2015b): The art of language invention. From Horse-Lords to Dark Elves, the Words Behind World-Building, Nueva York, Penguin Books.

Robles, José María (2016): «Así se crearon los idiomas de "Juego de Tronos" "Star Trek" y "Avatar" », El Mundo. En línea: <http://www.elmundo.es/papel/pantallas/2015/12/03/5660187246163f92368b457d.html> [26/02/2018].

SAlo, David (2004): A Gateway to Sindarin. A Grammar of an Elvish Language from J. R. R. Tolkien's Lord of the Rings, Salt Lake City, University of Utah Press.

Shippeys, Tom (2000): J. R. R. Tolkien: Author of the Century, Londres, HarperCollins.

Smith, Ross (2014): «Invented Languages and Writing Systems», en Stuart D. Lee (ed.), A Companion to J. R. R. Tolkien, John Wiley \& Sons, pp. 202-214.

TAYLOR, Cat A (2014): Inside HBO's Game of Thrones: Seasons 3 y 4, San Francisco, Chronicle Books. 
Tolkien, John R. R. (1965): El señor de los Anillos, Barcelona, Ediciones Minotauro.

Tolkien, John R. R. (1987): El Señor de los Anillos. Apéndices, Barcelona, Ediciones Minotauro. [Traducción de Rubén Masera].

Tolkien, J. R. R. (1993[1981]): Las cartas de J. R. R. Tolkien, Barcelona, Minotauro. [Traducción de Rubén Masera].

Tolkien, John R. R. (1998): Los monstruos y los criticos y otros ensayos, Barcelona, Minotauro.

Fecha de recepción: 8 de diciembre de 2018

Fecha de aceptación: 3 de mayo de 2019 
